\title{
On quotients and differences of hypergeometric functions
}

\author{
Slavko Simić ${ }^{*}$ and Matti Vuorinen ${ }^{2}$
}

\author{
* Correspondence: ssimic@turing. \\ mi.sanu.ac.rs \\ ${ }^{1}$ Mathematical Institute SANU, \\ Kneza Mihaila 36, 11000 Belgrade, \\ Serbia \\ Full list of author information is \\ available at the end of the article
}

\begin{abstract}
For Gaussian hypergeometric functions $F(x)=F(a, b ; c ; x), a, b, c>0$, we consider the quotient $Q_{F}(x, y)=(F(x)+F(y)) / F(z)$ and the difference $D_{F}(x, y)=F(x)+F(y)-F(z)$ for $0<x, y<1$ with $z=x+y-x y$. We give best possible bounds for both expressions under various hypotheses about the parameter triple $(a, b ; c)$.
\end{abstract}

2010 Mathematics Subject Classification: 26D06; 33C05.

Keywords: sub-additivity, hypergeometric functions, inequalities

\section{Introduction}

Among special functions, the hypergeometric function has perhaps the widest range of applications. For instance, several well-known classes of special functions such as complete elliptic integrals, Legendre functions, Chebyshev and Jacobi polynomials, and some elementary functions, such as the logarithm, are particular cases of it, cf. [1]. In a recent article [2] the authors studied various extensions of the Bernoulli inequality for functions of logarithmic type. In particular, the zero-balanced hypergeometric function $F(a, b ; a+b ; x), a, b>0$ occurs in these studies, because it has a logarithmic singularity at $x=1$, see (2.8) below. We now continue the discussion of some of the questions for quotients and differences of hypergeometric functions that were left open in [2].

Motivated by the asymptotic behavior of the function $F(x)=F(a, b ; c ; x)$ when $x \rightarrow 1^{-}$, see (2.8), we define for $0<x, y<1, a, b, c>0$

$$
Q_{F}(x, y):=\frac{F(x)+F(y)}{F(x+y-x y)}, \quad D_{F}(x, y):=F(x)+F(y)-F(x+y-x y) .
$$

Our task in this article is to give tight bounds for these quotients and differences assuming various relationships between the parameters $a, b, c$.

For the general case, we can formulate the following theorem.

Theorem 1.2. For $a, b, c>0$ and $0<x, y<1$ let $Q_{F}$ be as in (1.1). Then,

$$
0<Q_{F}(x, y) \leq 2
$$

The bounds in (1.3) are best possible as can be seen by taking $[1,15.1 .8]$

$$
F(x)=F(a, b ; b ; x)=(1-x)^{-a}:=F_{0}(x) .
$$

\section{Springer}


Then,

$$
Q_{F_{0}}(x, y)=\frac{(1-x)^{-a}+(1-y)^{-a}}{(1-x-y+x y)^{-a}}=\frac{(1-x)^{-a}+(1-y)^{-a}}{((1-x)(1-y))^{-a}}=(1-x)^{a}+(1-y)^{a},
$$

and the conclusion follows immediately. Similarly,

Theorem 1.4. For $a, b>0, c>a+b$ and $0<x, y<1$, we have

$$
\left|D_{F}(x, y)\right| \leq A
$$

with $A=A(a, b, c)=\frac{\Gamma(c) \Gamma(c-a-b)}{\Gamma(c-a) \Gamma(c-b)}=F(a, b ; c ; 1)$ as the best possible constant.

Most intriguing is the zero-balanced case. For example,

Theorem 1.6. For $a, b>0$ and $0<x, y<1$ let $D_{F}$ be as in (1.1). Then,

$$
\frac{R}{B}<D_{F}(x, y)<1
$$

with $R=R(a, b)=-2 \gamma-\psi(a)-\psi(b), B=B(a, b)$.

Both bounding constants are best possible.

In the sequel, we shall give a complete answer to an open question posed in [2].

\section{Preliminary results}

In this section, we recall some well-known properties of the Gaussian hypergeometric function $F(a, b ; c ; x)$ and certain of its combinations with other functions, for further applications.

It is well known that hypergeometric functions are closely related to the classical gamma function $\Gamma(x)$, the psi function $\psi(x)$, and the beta function $B(x, y)$. For $\operatorname{Re} x>0$, Re $y>0$, these functions are defined by

$$
\Gamma(x) \equiv \int_{0}^{\infty} e^{-t} t^{x-1} d t, \psi(x) \equiv \frac{\Gamma^{\prime}(x)}{\Gamma(x)}, B(x, y) \equiv \frac{\Gamma(x) \Gamma(y)}{\Gamma(x+y)},
$$

respectively (cf. [1, Chap. 6]). It is well known that the gamma function satisfies the difference equation $[1,6.1 .15]$

$$
\Gamma(x+1)=x \Gamma(x),
$$

and the reflection property $[1,6.1 .17]$

$$
\Gamma(x) \Gamma(1-x)=\frac{\pi}{\sin \pi x}=B(x, 1-x) .
$$

We shall also need the function

$$
R(a, b) \equiv-2 \gamma-\psi(a)-\psi(b), R(a) \equiv R(a, 1-a), R\left(\frac{1}{2}\right)=\log 16,
$$

where $\gamma$ is the Euler-Mascheroni constant given by

$$
\gamma=\lim _{n \rightarrow \infty}\left(\sum_{k=1}^{n} \frac{1}{k}-\log n\right)=0.577215 \ldots
$$


Given complex numbers $a, b$, and $c$ with $c \neq 0,-1,-2, \ldots$, the Gaussian hypergeometric function is the analytic continuation to the slit plane $\mathbb{C} \backslash[1, \infty)$ of the series

$$
F(a, b ; c ; z)={ }_{2} F_{1}(a, b ; c ; z)=\sum_{n=0}^{\infty} \frac{(a, n)(b, n)}{(c, n)} \frac{z^{n}}{n !}, \quad|z|<1 .
$$

Here $(a, 0)=1$ for $a \neq 0$, and $(a, n)$ is the shifted factorial function or the Appell symbol

$$
(a, n)=a(a+1)(a+2) \cdots(a+n-1)
$$

for $n \in \mathbb{N} \backslash\{0\}$, where $\mathbb{N}=\{0,1,2, \ldots\}$.

The hypergeometric function has the following simple differentiation formula ([1, 15.2.1])

$$
\frac{d}{d x} F(a, b ; c ; x)=\frac{a b}{c} F(a+1, b+1 ; c+1 ; x) .
$$

An important tool for our study is the following classification of the behavior of the hypergeometric function near $x=1$ in the three cases $a+b<c, a+b=c$, and $a+b>c$ :

$$
\left\{\begin{array}{l}
F(a, b ; c ; 1)=\frac{\Gamma(c) \Gamma(c-a-b)}{\Gamma(c-a) \Gamma(c-b)}, a+b<c \\
B(a, b) F(a, b ; a+b ; x)+\log (1-x)=R(a, b)+\mathrm{O}((1-x) \log (1-x)) \\
F(a, b ; c ; x)=(1-x)^{c-a-b} F(c-a, c-b ; c ; x), c<a+b .
\end{array}\right.
$$

Some basic properties of this series may be found in standard handbooks, see for example [1]. For some rational triples $(a, b, c)$, the functions $F(a, b ; c ; x)$ can be expressed in terms of well-known elementary function. A particular case that is often used in this article is $[1,15.1 .3]$

$$
g(x) \equiv x F(1,1 ; 2 ; x)=\log \frac{1}{1-x} .
$$

It is clear that for $a, b, c>0$ the function $F(a, b ; c ; x)$ is a strictly increasing map from $[0,1)$ into $[1, \infty)$ and that by $(2.8)$ we see that it is onto $[1, \infty)$ if $a+b \geq c$. For $a$, $b>0$ we see by $(2.8)$ that $x F(a, b ; a+b ; x)$ defines an increasing homeomorphism from $[0,1)$ onto $[0, \infty)$.

Theorem 2.10. [3],[[4], Theorem 1.52] For $a, b>0$, let $B=B(a, b)$ be as in (2.1), and let $R=R(a, b)$ be as in (2.4). Then the following are true.

(1) The function $f_{1}(x) \equiv \frac{F(a, b ; a+b ; x)-1}{\log (1 /(1-x))}$ is strictly increasing from $(0,1)$ onto $(a b /(a+$ b), $1 / B)$.

(2) The function $f_{2}(x) \equiv B F(a, b ; a+b ; x)+\log (1-x)$ is strictly decreasing from ( 0 , 1) onto $(R, B)$.

(3) The function $f_{3}(x) \equiv B F(a, b ; a+b ; x)+(1 / x) \log (1-x)$ is increasing from $(0,1)$ onto $(B-1, R)$ if $a, b \in(0,1)$.

(4) The function $f_{3}$ is decreasing from $(0,1)$ onto $(R, B-1)$ if $a, b \in(1, \infty)$. 
(5) The function

$$
f_{4}(x) \equiv \frac{x F(a, b ; a+b ; x)}{\log (1 /(1-x))}
$$

is decreasing from $(0,1)$ onto $(1 / B, 1)$ if $a, b \in(0,1)$.

(6) If $a, b>1$, then $f_{4}$ is increasing from $(0,1)$ onto $(1,1 / B)$.

(7) If $a=b=1$, then $f_{4}(x)=1$ for all $x \in(0,1)$.

We also need the following refinement of some parts of Theorem 2.10.

Lemma 2.11. [[5], Cor. 2.14] For $a, b>0$, let $B=B(a, b)$ be as in (2.1), and let $R=R$ $(a, b)$ be as in (2.4) and denote

$$
f(x) \equiv \frac{x F(a, b ; a+b ; x)}{\log (1 /(1-x))}
$$

(1) If $a \in(0, \infty)$ and $b \in(0,1 / a]$, then the function $f$ is decreasing with range $(1 / B, 1)$;

(2) If $a \in(1 / 2, \infty)$ and $b \geq a /(2 a-1)$, then $f$ is increasing from $(0,1)$ to the range

$(1,1 / B)$.

(3) If $a \in(0, \infty)$ and $b \in(0,1 / a]$, then the function $h$ defined by

$$
h(x):=B F(a, b ; a+b ; x)+(1 / x) \log (1-x)
$$

is increasing from $(0,1)$ onto $(B-1, R)$.

(4) If $a \in(1 / 3, \infty)$ and $b \geq(1+a) /(3 a-1)$, then $h$ is increasing from $(0,1)$ onto $(R, B-1)$.

For brevity, we write $\mathbb{R}_{+}=(0, \infty)$.

Lemma 2.12. (Cf. $[4,1.24,7.42(1)])$ (1) If $E(t) / t$ is an increasing function on $\mathbb{R}_{+}$, then $E$ is sub-additive, i.e., for each $x, y>0$ we have that

$$
E(x)+E(y) \leq E(x+y) .
$$

(2) If $E(t) / t$ decreases on $\mathbb{R}_{+}$, then $E$ is a super-additive function, that is

$$
E(x)+E(y) \geq E(x+\gamma)
$$

for $x, y \in \mathbb{R}_{+}$.

\section{Main results}

By (2.8), the zero-balanced hypergeometric function $F(a, b ; a+b ; x)$ has a logarithmic singularity at $x=1$. We shall now demonstrate that its behavior is nearly logarithmic also in the sense that some basic identities of the logarithm yield functional inequalities for the zero-balanced function.

Next, writing the basic addition formula for the logarithm

$$
\log z+\log w=\log (z w), z, w>0,
$$

in terms of the function $g$ in (2.9), we have

$$
g(x)+g(y)=g(x+y-x y), x, y \in(0,1) .
$$


Based on this observation and a few computer experiments, we posed in [2] the following question:

Question 3.1. Fix $c, d>0$ and let $g(x)=x F(c, d ; c+d ; x)$ for $x \in(0,1)$ and set

$$
Q_{g}(x, y)=\frac{g(x)+g(y)}{g(x+y-x y)}
$$

for $x, y \in(0,1)$.

(1) For which values of $c$ and $d$, this function is bounded from below and above?

(2) Is it true that

a) $Q_{g}(x, y) \geq 1$, if $c d \leq 1$ ?

b) $Q_{g}(x, y) \leq 1$, if $c, d>1$ ?

c) Are there counterparts of Theorem 1.6 for the function

$$
D_{g}(x, y)=g(x)+g(y)-g(x+y-x y) ?
$$

We shall give a complete answer to this question in the sequel.

Note first that the quotient $Q_{g}$ is always bounded. Namely,

Theorem 3.2. For all $c, d>0$ and all $x, y \in(0,1)$ we have that

$$
0<Q_{g}(x, y)<2 .
$$

A refinement of these bounds for some particular $(c, d)$ pairs is given by the following two assertions.

Theorem 3.3. (1) For $c, d>0, c d \leq 1$ and $x, y \in(0,1)$ we have

$$
\frac{1}{B(c, d)} \leq Q_{g}(x, y) \leq B(c, d)
$$

(2) For $c, d>0,1 / c+1 / d \leq 2$ and $x, y \in(0,1)$ we have

$$
B(c, d) \leq Q_{g}(x, y) \leq \frac{1}{B(c, d)} .
$$

Note that parts (1) and (3) of Lemma 2.11 imply that for $c, d>0, c d \leq 1,(c, d) \neq(1,1)$ we have

$$
R(c, d)>0, B(c, d)>1 .
$$

Theorem 3.5. For $c d \leq 1$ and $x, y \in(0,1)$ we have

$$
\frac{B(c, d)-1}{R(c, d)} \leq Q_{g}(x, y) \leq \frac{2 R(c, d)}{B(c, d)-1} .
$$

We shall prove now the hypothesis from the second part of Question 3.1 under the condition $1 / c+1 / d \leq 2$ in part (b) which, in particular, includes the case $c>1, d>1$.

Theorem 3.6. Fix c, $d>0$ and let $Q$ and $g$ be as in Question 3.1.

(1) If $c d \leq 1$ then $Q_{g}(x, y) \geq 1$ for all $x, y \in(0,1)$.

(2) If $1 / c+1 / d \leq 2$, then $Q_{g}(x, y) \leq 1$ for all $x, y \in(0,1)$.

Counterparts of Theorem 1.6 for the difference $D_{g}$ are given in the next assertion. 
Theorem 3.7. Fix c, $d>0$ and let $D$ be as in Question 3.1.

(1) If $c d \leq 1$, then

$0 \leq D_{g}(x, y)<\frac{2 R(c, d)+1}{B(c, d)}-1$

for all $x, y \in(0,1)$.

(2) If $1 / c+1 / d \leq 2$, then

$$
\frac{2 R(c, d)+1}{B(c, d)}-1<D_{g}(x, y) \leq 0
$$

for all $x, y \in(0,1)$.

Combining the results above, we obtain the following two-sided bounds for the quotient $Q_{g}$.

Corollary 3.8. Fix c, $d>0$ and let $Q$ be as in Question 3.1.

(1) If $c d \leq 1$, then

$$
1 \leq Q_{g}(x, y)<\min \{B(c, d), 2\}
$$

for all $x, y \in(0,1)$.

(2) If $1 / c+1 / d \leq 2$, then

$$
B(c, d)<Q_{g}(x, y) \leq 1
$$

for all $x, y \in(0,1)$.

The assertions above represent a valuable tool for estimating quotients and differences of a hypergeometric function with different arguments. To illustrate this point, we give an example.

In [2], motivated by the relation $g(x)=2 g(1-\sqrt{1-x})$ with $g$ as in (2.9), the authors asked the question about the bounds for the function $S(t)$ defined by

$$
S(t):=\frac{g(t)}{g(1-\sqrt{1-t})}, t \in(0,1),
$$

where $g(t):=t F(a, b ; a+b ; t), a, b>0$.

An answer follows instantly applying Corollary 3.8.

Theorem 3.9. $\operatorname{Let} S(t):=\frac{g(t)}{g(1-\sqrt{1-t})}, t \in(0,1)$, with $g(t):=t F(a, b ; a+b ; t), a, b>0$.

(1) If $a b \leq 1$, then

$$
1<S(t) \leq 2 .
$$


(2) If $1 / a+1 / b \leq 2$, then

$$
2 \leq S(t)<\frac{2}{B(a, b)}
$$

\section{Proofs}

\subsection{Proof of Theorem 1.2}

The proof is based solely on the monotonicity property of the function $F(x)=F(a, b ; c$;

$x)$. Namely, for $x, y \in(0,1)$, put $z=x+y-x y, z \in(0,1)$. Since

$$
x \leq \max \{x, y\}, y \leq \max \{x, y\} ; z \geq \max \{x, y\},
$$

and $F(u)$ is monotone increasing in $u$, we obtain

$$
Q_{F}(x, y)=\frac{F(x)+F(y)}{F(z)} \leq \frac{2 F(\max \{x, y\})}{F(\max \{x, y\})}=2 .
$$

The left-hand bound is trivial. $\square$

\subsection{Proof of Theorem 1.4}

The assertion of this theorem is a consequence of the previous one and (2.8). Indeed, from (1.3) we get

$$
-F(a, b ; c ; z)<D_{F}(x, y) \leq F(a, b ; c ; z),
$$

that is,

$$
\left|D_{F}(x, y)\right| \leq F(a, b ; c ; z)=F(a, b ; c ; 1-(1-x)(1-y)) \leq F(a, b ; c ; 1)=A .
$$

$\square$

\subsection{Proof of Theorem 1.6}

Consider the function

$$
s(x)=F(a, b, a+b ; x)-F(a, b, a+b ; x+y-x y),
$$

where $y, y \in(0,1)$, is an independent parameter.

Since

$$
\begin{gathered}
s^{\prime}(x)=F^{\prime}(a, b, a+b ; x)-(1-y) F^{\prime}(a, b, a+b ; x+y-x y) \\
=\frac{a b}{a+b}(F(a+1, b+1, a+b+1 ; x)-(1-y) F(a+1, b+1, a+b+1 ; x+y-x y))
\end{gathered}
$$

We get $(1-x) s^{\prime}(x)$

$$
\begin{gathered}
=\frac{a b}{a+b}((1-x) F(a+1, b+1, a+b+1 ; x)-(1-x)(1-y) F(a+1, b+1, a+b+1 ; x+y-x y)) \\
=\frac{a b}{a+b}(F(a, b, a+b+1 ; x)-F(a, b, a+b+1 ; x+y-x y))<0 .
\end{gathered}
$$

Therefore, $s(x)$ is monotone decreasing on $(0,1)$ and, consequently,

$$
s(x)<\lim _{x \rightarrow 0^{+}} s(x)=1-F(a, b, a+b ; y) .
$$


Also, by Theorem 2.10, part 2, we obtain

$$
s(x)>\lim _{x \rightarrow 1^{-}} s(x)=\frac{1}{B} \log (1-y)>\frac{R}{B}-F(a, b, a+b ; y) .
$$

Since $D_{F}\left(0^{+}, 0^{+}\right)=1$ and $D_{F}\left(1^{-}, 1^{-}\right)=R / B$, cited bounds are best possible. $\square$

\subsection{Proof of Theorem 3.2}

Analogously to the proof of Theorem 1.2, we have

$$
Q_{g}(x, y)=\frac{x F(x)+y F(y)}{z F(z)} \leq \frac{(x+y) F(\max \{x, y\})}{z F(\max \{x, y\})}=\frac{x+y}{z}<2 .
$$

$\square$

\subsection{Proof of Theorem 3.3}

Lemma 2.11 (1) yields

$$
\frac{1}{B} \log (1 /(1-u)) \leq u F(u) \leq \log (1 /(1-u)),
$$

for $u \in(0,1), c d \leq 1$.

Therefore,

$$
\frac{x F(x)+y F(y)}{(x+y-x y) F(x+y-x y)} \leq \frac{\log \frac{1}{1-x}+\log \frac{1}{1-y}}{\frac{1}{B} \log \frac{1}{(1-x)(1-y)}}=B(c, d) .
$$

The lower bound is proved in the same way.

Applying part (2) of Lemma 2.11, we bound $Q_{g}$ similarly in the case $1 / c+1 / d \leq 2$. $\square$ Remark 4.1. From parts (1) and (3) of Lemma 2.11, we conclude that

$$
R(c, d)>B(c, d)-1>0,
$$

for $c, d>0, c d \leq 1,(c, d) \neq(1,1)$.

\subsection{Proof of Theorem 3.4}

Let us write $B=B(c, d), R=R(c, d)$ and $L=\log (1 /((1-x)(1-y)))>0$. By Lemma 2.11

(3) we have

$$
\frac{B-1}{B} x+\frac{1}{B} \log \frac{1}{1-x}<x F(c, d ; c+d ; x)<\frac{R x}{B}+\frac{1}{B} \log \frac{1}{1-x} .
$$

Since $x+y<2(x+y-x y)$ we obtain by $(4.2)$

$$
Q_{g}(x, y) \leq \frac{\frac{R(x+y)}{B}+\frac{L}{B}}{\frac{B-1}{B}(x+y-x y)+\frac{L}{B}} \leq \frac{2 R(x+y-x y)+L}{(B-1)(x+y-x y)+L} \leq \frac{2 R}{B-1},
$$

and

$$
Q_{g}(x, y) \geq \frac{(B-1)(x+y)+L}{R(x+y-x y)+L} \geq \frac{(B-1)(x+y-x y)+L}{R(x+y-x y)+L} \geq \frac{B-1}{R} .
$$

口 


\subsection{Proof of Theorem 3.6}

By the first part of Lemma 2.11, $f$ is monotone decreasing for $c d \leq 1$.

Hence, for $0<x<y<1$ we have

$$
\frac{x F(c, d ; c+d ; x)}{\log (1 /(1-x))} \geq \frac{y F(c, d ; c+d ; y)}{\log (1 /(1-\gamma))} .
$$

Putting $1-x=e^{-u}, 1-y=e^{-v} ; u, v \in(0, \infty)$, we get that the inequality

$$
\frac{\left(1-e^{-u}\right) F\left(c, d ; c+d ;\left(1-e^{-u}\right)\right)}{u} \geq \frac{\left(1-e^{-v}\right) F\left(c, d ; c+d ;\left(1-e^{-v}\right)\right)}{v}
$$

holds whenever $0<u<v<\infty$.

This means that the function $G(t) / t$ is monotone decreasing, where

$$
G(t):=\left(1-e^{-t}\right) F\left(c, d ; c+d ;\left(1-e^{-t}\right)\right)=g\left(1-e^{-t}\right) .
$$

By Lemma 2.12, it follows that $G$ is super-additive, that is

$$
G(u)+G(v) \geq G(u+v),
$$

which is equivalent to

$$
g(x)+g(y) \geq g(x+y-x y),
$$

and the proof of the first part of Theorem 3.6 is complete.

The proof of the second part is similar. Note that the condition $c \in(1 / 2, \infty), d \geq c /$ $(2 c-1)$ of Lemma 2.11 is equivalent to the condition $1 / c+1 / d \leq 2$ of Theorem 3.6.

\subsection{Proof of Theorem 3.7}

(1) The left-hand side of this inequality is a direct consequence of part (1) of Theorem 3.6.

Next, from Lemma 2.11, part (3) for $u \in(0,1), c d \leq 1$, we get

$$
(B-1) u-\log (1-u)<B u F(u)<R u-\log (1-u) .
$$

Hence, by the terminology from Theorem 3.2, we obtain

$$
\begin{gathered}
B D_{g}(x, y)=B x F(x)+B y F(y)-B z F(z)<\log (1-z)-\log (1-x)-\log (1-y)+R(x+y)-(B-1) z \\
=(R-B+1)(x+y)+(B-1) x y<2(R-B+1)+(B-1)=2 R+1-B
\end{gathered}
$$

since Remark 4.1 yields $R-B+1>0$ and $B-1>0$.

(2) To prove this part we shall use Lemma 2.11, part (4). Because $d \geq c /(2 c-1) \geq$ $(c+1) /(3 c-1)$ and $(1 / 2, \infty) \subset(1 / 3, \infty)$, we conclude that this assertion is valid under the condition $1 / c+1 / d \leq 2$.

Therefore, for $u \in(0,1), 1 / c+1 / d \leq 2,(c, d) \neq(1,1)$, we get

$$
R u-\log (1-u)<B u F(u)<(B-1) u-\log (1-u),
$$


and, as above,

$$
\begin{aligned}
& B D_{g}(x, y)=B x F(x)+B y F(y)-B z F(z)>\log (1-z)-\log (1-x)-\log (1-\gamma)+R(x+\gamma)-(B-1) z \\
& =(R-B+1)(x+y)+(B-1) x y>2(R-B+1)+(B-1)=2 R+1-B,
\end{aligned}
$$

since parts (1) and (4) of Lemma 2.11 give $R-B+1<0$ and $B-1<0$.

Because the right-hand inequality is a consequence of Theorem 3.6, part (2), the proof is complete.

\subsection{Proof of Theorem 3.9}

Putting $x=y=1-\sqrt{1-t}$, we obtain $z=x+y-x y=t$. Therefore,

$$
Q_{g}(x, y)=2 / S(t) \text {. }
$$

The rest is an application of Corollary 3.8. $\square$

\section{Acknowledgements}

The authors are indebted to the referee for his/her constructive comments. The research of Matti Vuorinen was supported by the Academy of Finland, Project 2600066611. This project also supported Slavko Simić' visit to Finland.

\section{Author details}

${ }^{1}$ Mathematical Institute SANU, Kneza Mihaila 36, 11000 Belgrade, Serbia ${ }^{2}$ Department of Mathematics, University of

Turku, 20014 Turku, Finland

\section{Authors' contributions}

Some of the main problems in this paper were motivated by earlier work and computer experiments of MV. SS found most proofs. All authors read and approved the final manuscript.

\section{Competing interests}

The authors declare that they have no competing interests.

Received: 15 July 2011 Accepted: 21 December 2011 Published: 21 December 2011

\section{References}

1. Abramowitz M, Stegun IA (eds.): Handbook of Mathematical Functions with Formulas, Graphs and Mathematical Tables. Dover, New York. (1965)

2. Klén, R, Manojlović, V, Simić, S, Vuorinen, M: Bernoulli inequality and hypergeometric functions, 15 pp. arXiv math.CA 1106.1768. (2011)

3. Anderson, GD, Barnard, RW, Richards, KC, Vamanamurthy, MK, Vuorinen, M: Inequalities for zero-balanced hypergeometric functions. Trans Am Math Soc. 347, 1713-1723 (1995). doi:10.2307/2154966

4. Anderson, GD, Vamanamurthy, MK, Vuorinen, M: Conformal Invariants, Inequalities and Quasi-conformal Maps. John Wiley \& Sons, New York. (1997)

5. Ponnusamy, S, Vuorinen, M: Asymptotic expansions and inequalities for hypergeometric functions. Mathematika. 44, 278-301 (1997). doi:10.1112/S0025579300012602

doi:10.1186/1029-242X-2011-141

Cite this article as: Simić and Vuorinen: On quotients and differences of hypergeometric functions. Journal of Inequalities and Applications 2011 2011:141.

\section{Submit your manuscript to a SpringerOpen ${ }^{\circ}$} journal and benefit from:

- Convenient online submission

- Rigorous peer review

- Immediate publication on acceptance

- Open access: articles freely available online

- High visibility within the field

- Retaining the copyright to your article

Submit your next manuscript at $\boldsymbol{s p r i n g e r o p e n . c o m ~}$ 